\title{
Una comparativa normativa - experimental sobre el hormigón reforzado con fibras sometido a esfuerzo de corte directo
}

\author{
A. Picazo ${ }^{1}$, M.G. Alberti², A. Enfedaque ${ }^{2}$, J.C. Gálvez ${ }^{2 *}$ \\ *Autor de Contacto: jaime.galvez@upm.es \\ ${ }^{1}$ Departamento de Tecnología de la Edificación. E.T.S. de Edificación. Universidad Politécnica de Madrid. Avda. \\ Juan de Herrera 6, 28040 Madrid-España. \\ ${ }^{2}$ Departamento de Ingeniería Civil: Construcción. E.T.S. de Ingenieros de Caminos, Canales y Puertos. Universidad \\ Politécnica de Madrid. C/ Profesor Aranguren, s/n, 28040 Madrid-España.
}

\section{RESUMEN}

La normativa de hormigón estructural permite considerar el carácter estructural del hormigón reforzado con fibras (HRF) en función de resistencias residuales obtenidas en ensayos de resistencia a la tracción por flexión. Sin embargo, en relación con la fractura a cortante, todavía no hay consenso en el ámbito de la investigación sobre si es apropiado el uso de resistencias a tracción en la formulación para la resistencia frente a solicitaciones de cortante. Mediante el empleo de fibras es posible reducir el refuerzo de barras de acero y, en el caso de cortante, llegar a eliminar la armadura. Con la finalidad de profundizar en el comportamiento del HRF sometido a cortante, se desarrolló una campaña experimental con HRF con fibras de acero y poliolefina sometido a esfuerzos de cortante puro mediante ensayos "push-off". Las probetas ensayadas procedían de ensayos previos de flexión, por lo que se conocían sus resistencias residuales a flexión. Estas resistencias se compararon con los ensayos a fractura en cortante concluyéndose que los valores normativos resultaban muy conservadores para pequeños deslizamientos a cortante. $\mathrm{Al}$ aumentar el deslizamiento los valores de resistencia normativos y experimentales resultaron parecidos, e incluso para deslizamientos grandes los valores experimentales resultaron superiores los normativos.

Palabras clave: esfuerzo cortante, hormigón reforzado con fibras, ensayo "push-off", fibras de acero, fibras de poliolefina 
The structural concrete standards allow to use the fibres in the design of structural concrete, performing the fibre reinforced concrete (FRC). The residual strengths used for design are obtained from bending tests. However, regarding to shear fracture, there is still no consensus in the field of research about how to use tensile strengths in the formulation for shear stress design. Through the use of fibres, it is possible to reduce the reinforcement with steel bars and, in the case of shear, even to avoid the reinforcement. In order to deepen the behaviour of FRC subjected to shear, an experimental campaign was developed with concrete reinforced with steel and polyolefin fibres subjected to pure shearing efforts through push-off tests. The tested specimens came from previous bending tests, so their residual resistances in bending were known. These resistances were compared with the fracture tests on shear, concluding that the normative values were very conservative for small shear slips. As the slippage increased, the normative and experimental resistance values were similar, and even for large slides the experimental values were higher than the normative ones.

Keywords: shear strength, fibre reinforced concrete, push-off test, steel fibres, polyolefin fibres

\section{INTRODUCCIÓN}

El uso de fibras como refuerzo del hormigón evita la fragilidad del material, dotándole de ductilidad (di Prisco et al., 2009). Además, mejora sus características mecánicas, consigue controlar la abertura de fisuras y aumentar la energía de fractura (Bencardino et al., 2010). Éste refuerzo de fibras permite sustituir de manera parcial el refuerzo de barras de acero a flexión (You et al., 2011). En el caso de esfuerzos de cortante, el refuerzo con fibras puede sustituir al refuerzo de cercos (Yoo et al., 2017). Así, el HRF es ampliamente empleado en dovelas de túneles (Conforti et al., 2019), canalizaciones (Alberti et al., 2017), losas alveolares (Conforti et al., 2020), pasarelas peatonales (López et al., 2014) y estructuras de cubiertas (Cabo et al., 2003), entre otras. Para poder tener en cuenta la contribución estructural del refuerzo mediante fibras, las normas exigen una serie requerimientos basados en los resultados de fractura en modo I. Es decir, para cualquier tipo de esfuerzo al que esté sometida la estructura, las capacidades mecánicas se obtienen en función de resistencias residuales a tracción por flexión obtenidas de manera experimental sobre vigas a flexión en tres o cuatro puntos con entalla (International Federation for Structural Concrete (fib), 2010, Ministerio de Fomento, 2008).

El empleo de tensiones residuales a flexo-tracción para el cálculo de refuerzos de cortante muestra falta de unanimidad, por lo que es de interés su investigación. De esta forma, nos encontramos con investigaciones dispares. Así, en la referencia (Krassowska et al., 2019) analizaron vigas de hormigón reforzadas, indicando el buen ajuste de la formulación del CM-2010 (International Federation for Structural Concrete (fib), 2010). De forma similar, en (Ortiz-Navas et al., 2020) mediante ensayos sobre vigas de hormigón con fibras de polipropileno y acero, concluyeron que el empleo de tensiones residuales de flexo-tracción son apropiadas para la caracterización de piezas estructurales a cortante. Sin embargo, en la referencia (Cuenca et al., 2015) concluyeron, en su estudio sobre vigas, la necesidad de revisar la formulación a cortante en función de las características de las fibras. Usando fibras híbridas y macrosintéticas en vigas, en la referencia (Dev et al., 2020) indicaron que la formulación de la ACI y RILEM resultaba conservadora. Por último, sobre losas alveolares, los resultados han mostrado (Conforti et al., 2020) la necesidad de ajustar dicha formulación.

El presente estudio aborda el estudio del comportamiento a fractura en cortante de varios tipos de hormigón en referencia a las normas CM-2010 (International Federation for Structural Concrete 
(fib), 2010) y EHE-08 (Ministerio de Fomento, 2008). Para ello se emplearon probetas tipo pushoff con cuatro tipologías de hormigón: un hormigón autocompactante de alta resistencia a compresión (60 MPa) reforzado con fracciones volumétricas de $0,64 \%$ y $0,89 \%$ de fibras de acero. El segundo es un hormigón de moderada resistencia a compresión (40 MPa) y fracción volumétrica de $1,10 \%$ de fibras de poliolefina. Un tercer hormigón, de similares características al segundo, pero autocompactante e igualmente reforzado con un $1,10 \%$ de fibras de poliolefina. Y el cuarto hormigón, de baja resistencia (20 MPa) reforzado con fracciones volumétricas de $0,66 \%$ y $0,83 \%$ de fibras de poliolefina.

Los diferentes hormigones fueron previamente ensayados a flexión (Alberti et al., 2015, Alberti et al., 2019, Picazo, 2020), superando todos ellos los requerimientos estructurales del MC-2010 y la EHE-08 para poder considerar la contribución resistente de las fibras en el cálculo: $\left(f_{R I} / f_{L O P}>0,4 \mathrm{y}\right.$ $\left.f_{R 3 k} / f_{R I k}>0,5\right)$.

\section{MATERIALES Y PROPIEDADES MECÁNICAS DE LOS HORMIGONES}

La dosificación empleada en la realización de los hormigones se puede ver en investigaciones previas (Picazo, 2020). Es destacable indicar la diferencia de cantidad de cemento empleado en la realización de los hormigones, lo que da lugar a tres tipologías de hormigón en relación a su resistencia a compresión. Para los hormigones de baja resistencia se empleó cemento EN 197-1 CEM II 32,5 B-M, mientras que para el resto de hormigones se empleó un EN 197-1 CEM I 52,5 R-SR 5 con adición de filler calizo y superplastificante con base de policarboxilato. En todos los casos el tamaño máximo del árido fue de $12,7 \mathrm{~mm}$.

Las fibras de poliolefina empleadas tenían acabado rugoso, para facilitar la adherencia, y una resistencia a tracción de $1100 \mathrm{~N} / \mathrm{mm}^{2}$. Se emplearon con una longitud de $60 \mathrm{~mm}$ en los hormigones con fracción volumétrica de $1,10 \%$ y de $48 \mathrm{~mm}$ en los hormigones de baja resistencia y fracciones volumétricas de $0,66 \%$ y $0,83 \%$. Con respecto a las fibras de acero lisas, con extremos conformados en patillas, se utilizaron de tipología RC80/50BN en el hormigón de fracción volumétrica de $0,64 \%$ y una mezcla al 50\% de éstas con otras de tipo RC65/35BN en el hormigón de mayor fracción volumétrica $(0,89 \%)$. Las características de las fibras se pueden consultar en investigaciones previas (Picazo et al., 2018).

De esta forma se realizaron seis hormigones designados como SCS70 y SCS50, autocompactantes de alta resistencia a compresión y reforzados con $0,64 \%$ y $0,89 \%$ de fibras de acero. Los VP6 y VP75, compactados por vibración, de baja resistencia y reforzados con $0,66 \%$ y $0,83 \%$ de fibras de poliolefina. Por último, SCP10 y VP10, reforzados con un $1,10 \%$ de fibras de poliolefina, autocompactante y vibrado, respectivamente.

Los resultados de los ensayos previos para obtener las resistencias a compresión $\left(f_{c m}\right)$ y las capacidades mecánicas y tensiones residuales a tracción por flexión $\left(f_{L O P}, f_{R 1}\right.$ y $\left.f_{R 3}\right)$ en ensayos conforme a la norma EN14651:2007+A1 (European Committee for Standardization, 2007) se muestran en la Tabla 1.

Tabla 1. Resistencias a compresión y tensiones residuales por flexión (MPa) de los hormigones estudiados. 


\begin{tabular}{|c|c|c|c|c|}
\hline & $\boldsymbol{f}_{\boldsymbol{c m}}$ & $\boldsymbol{f}_{\boldsymbol{L} \boldsymbol{O P}}$ & $\boldsymbol{f}_{\boldsymbol{R} \boldsymbol{1}}$ & $\boldsymbol{f}_{\boldsymbol{R} 3}$ \\
\hline SCS50 & 62,2 & 5,69 & 7,29 & 5,27 \\
\hline SCS70 & 58,8 & 6,50 & 11,08 & 9,46 \\
\hline $\mathbf{V P 6}$ & 21,7 & 2,76 & 1,43 & 1,75 \\
\hline VP75 & 20,1 & 2,75 & 1,62 & 1,97 \\
\hline SCP10 & 51,5 & 5,22 & 2,41 & 3,87 \\
\hline VP10 & 39,7 & 4,21 & 1,98 & 2,87 \\
\hline
\end{tabular}

\section{PROGRAMA EXPERIMENTAL DE ENSAYOS A CORTANTE PUSH- OFF}

Las probetas tipo push-off tenían forma prismática con unas dimensiones de 270x150x150 mm $\mathrm{mm}^{3}$ Fueron obtenidas de las mitades residuales de ensayos a flexión, debidamente mecanizadas y eliminando la zona afectada por el ensayo de tracción por flexión. Como se puede observar en la Figura 1(a), en dicho prisma se realizaron dos entallas de $75 \mathrm{~mm}$ de profundidad y $9 \mathrm{~mm}$ de espesor. Mediante la realización de estas entallas la sección de ligamento sometida a cortante tuvo unas medidas de $150 \mathrm{~mm}$ de ancho y $75 \mathrm{~mm}$ de altura. Es importante indicar que las probetas se realizaron para los ensayos de flexión por lo que el posible posicionamiento de las fibras debe resultar paralelo a la dirección de carga en los ensayos push-off. De tal forma, la disposición de las fibras no es la mejor para soportar los esfuerzos de cortante, por lo que los resultados pueden considerarse como conservadores.

(a)

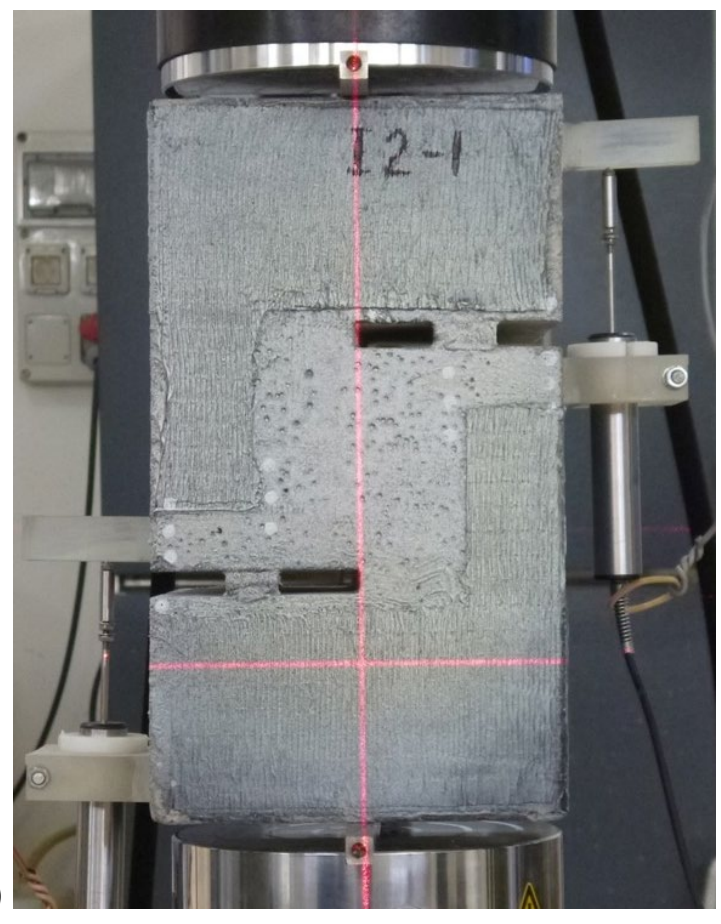

(b)

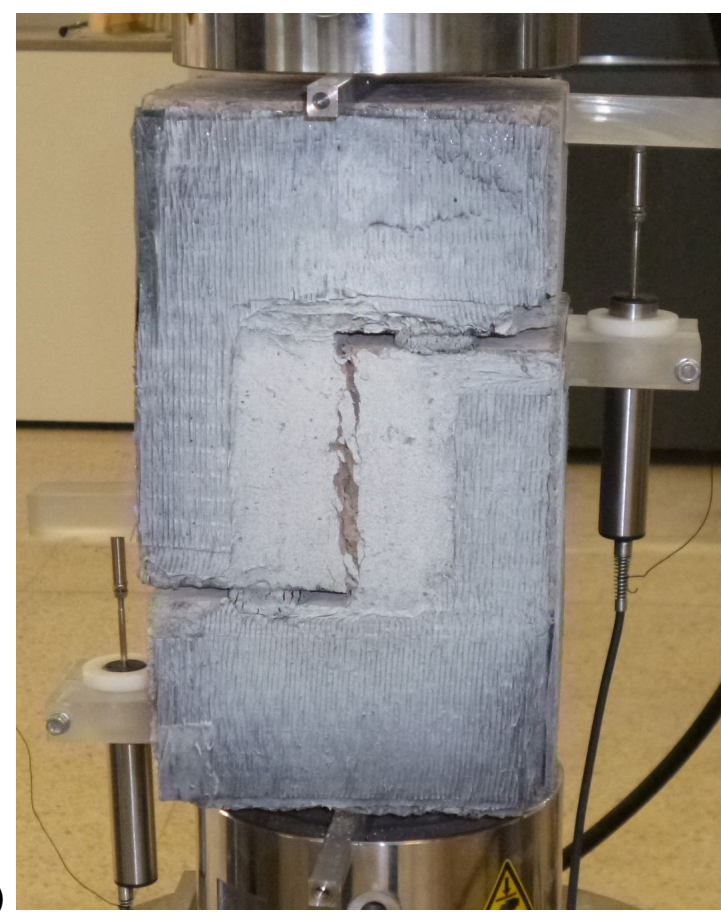

Figura 1. (a) Probeta push-off preparada para ser ensayada y (b) ensayada.

Para el correcto funcionamiento del ensayo push-off se colocaron las probetas de forma especialmente cuidadosa. Las barras de carga metálicas superior e inferior, de dimensiones 200x10x10 mm $\mathrm{mm}^{3}$ se posicionaron haciendo coincidir su centro con la sección de ligamento, cosa que se consiguió mediante un nivel láser. Se dispusieron, en ambas entallas, dos transductores de 
desplazamiento lineal variable (LVDT) para medir la abertura o cierre de las puntas de las entallas $\mathrm{y}$, como media, obtener los deslizamientos a cortante en la sección de ligamento. De tal forma, la Figura 1(b) muestra una probeta tras ser ensayada. Los ensayos se realizaron en una máquina Instron ${ }^{\circledR}$, con una capacidad de carga de $500 \mathrm{kN}$, siendo controlados por desplazamiento del actuador a una velocidad de $1 \mu \mathrm{m} / \mathrm{s}$.

\section{RESULTADOS Y DISCUSIÓN}

\subsection{Contribución de las fibras en el HRC sometido a cortante}

Con el fin de comparar los hormigones estudiados se consideraron las diferentes tensiones a cortante durante los ensayos como resultado de aplicar la ecuación (1), dividiendo la carga aplicada (L) entre la superficie de la sección de ligamento (S). De tal forma se obtuvieron tensiones medias, independiente de la sección resistente.

$$
\tau_{\text {aver }}=L / S
$$

La Tabla 2 muestra el número de probetas ensayadas de cada hormigón, su tensión rasante promedio máxima $\left(\tau_{M A X}\right)$, la carga máxima media soportada $\left(L_{M A X}\right)$ y el número de fibras contadas en la sección de ligamento tras los ensayos.

Para estudiar el comportamiento de los diferentes HRC en los ensayos push-off es importante estudiar la Figura 2. Ésta muestra la gráfica tensión media a cortante frente a deslizamiento a cortante. En todos los casos el comportamiento general es el mismo, una rama de carga hasta el primer punto de inflexión donde, tras producirse la fisuración de las probetas, se produjo una rama de descarga más o menos pronunciada. Posteriormente se puede observar una rama de tensión descendente, pero con mínima inclinación, correspondiendo a la fase residual de los ensayos, gobernada por la actuación como refuerzo de las fibras.

Tabla 2. Resultados de los ensayos de cortante tipo push-off.

\begin{tabular}{|c|c|c|c|c|}
\hline & $\begin{array}{c}\text { Prob. } \\
(\mathbf{u d s})\end{array}$ & $\begin{array}{c}\tau_{\text {MAX }} \\
(\mathbf{M P a})\end{array}$ & $\begin{array}{c}\boldsymbol{L}_{\text {MAX }} \\
(\mathbf{k N})\end{array}$ & $\begin{array}{c}\text { Fibras } \\
(\mathbf{u d s})\end{array}$ \\
\hline SCS50 & 2 & 12,2 & 131,4 & 82 \\
\hline SCS70 & 2 & 17,4 & 141,9 & 116 \\
\hline VP6 & 4 & 5,2 & 65,0 & 63 \\
\hline VP75 & 4 & 4,9 & 68,7 & 89 \\
\hline SCP10 & 3 & 9,7 & 105,0 & 69 \\
\hline VP10 & 2 & 7,9 & 99,8 & 79 \\
\hline
\end{tabular}

Los hormigones pueden estudiarse agrupados de dos en dos, función de su matriz cementicia. Así el SCS50 y SCS70 presentan una rama de carga más pronunciada que el resto de los hormigones. En ambos hormigones se dio la circunstancia de que la primera fisura se produjo antes del punto de inflexión de carga máxima, por lo que dichos hormigones, tras fisurarse siguieron aumentando su capacidad resistente a cortante. Entre el SCS50 (12,2 MPa) y el SCS70 (17,4 MPa), con la misma matriz, se aprecia un aumento de resistencia del 42,6\% en su valor máximo. En el resto de los hormigones la matriz se fisuró en el punto de máxima resistencia mostrado en la Figura 2. 


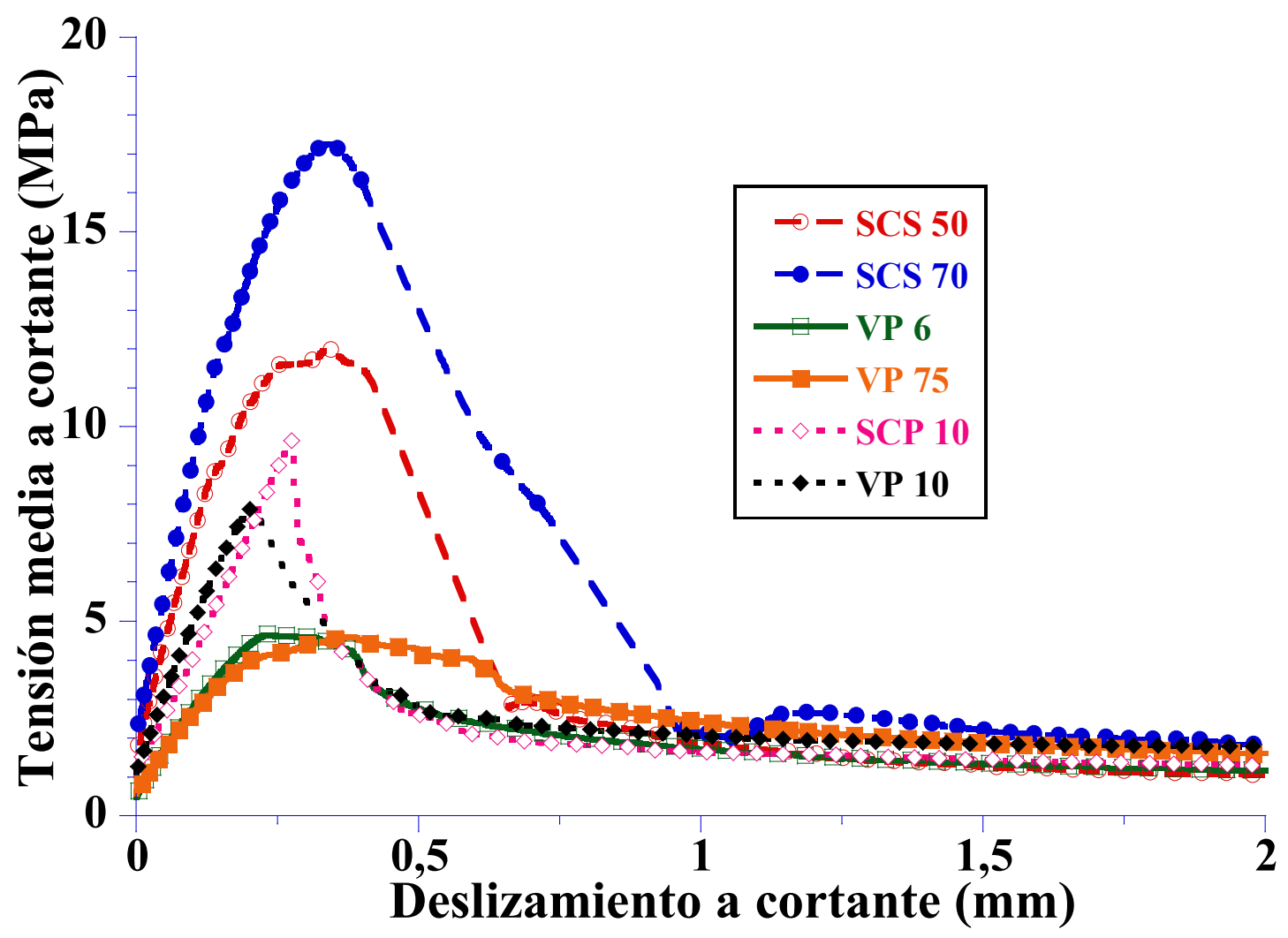

Figura 2. Curvas medias tensión - deslizamiento a cortante en los ensayos push-off. Se muestra la gráfica post-fisuración solamente hasta $2 \mathrm{~mm}$ de deslizamiento.

La diferencia de inclinación de los hormigones es función de la matriz de hormigón sin fisurar, por lo que se puede ver que las gráficas de los hormigones reforzados con fibras de acero, y mejor matriz, muestran una inclinación mayor. El siguiente grupo serían los hormigones reforzados con $10 \mathrm{~kg} / \mathrm{m}^{3}$ de fibras de poliolefina. Por último, los hormigones de baja resistencia a compresión mostraron una menor inclinación en la rama de carga.

Tras el punto de inflexión correspondiente al valor de tensión máxima cortante los hormigones SCS50 y SCS70 mostraron una caída brusca de carga asociada a un deslizamiento elevado entre las dos caras de la sección de ligamento. En el caso de los VP6 y VP75 se produjo un descenso en el valor de la tensión, pero mostrando una gran ductilidad, sin caídas bruscas de carga, tal y como se aprecia en la Figura 2 con unas ramas de descarga con baja inclinación. Por último, tanto en el SCP10 como en el VP10, su comportamiento fue intermedio a lo comentado: se produjo una rama de descarga brusca, pero no asociada a un gran deslizamiento.

Una vez se llegó, en todos los hormigones, a un deslizamiento de $1 \mathrm{~mm}$ los valores de resistencias residuales se igualaron en todos los casos, con valores que oscilaron entre 1,6 y $2,5 \mathrm{~N} / \mathrm{mm}^{2}$. En este punto es cuando la gráfica de la rama residual muestra un comportamiento función de las fibras contadas en las diferentes secciones de fractura.

\subsection{Resultados experimentales vs numéricos normativos a cortante}


Investigaciones previas (Conforti et al., 2020, Ortiz-Navas et al., 2020, Dev et al., 2020) difieren respecto a si la formulación adoptada por las normativas de diseño estructural de hormigón armado es apropiada para el cálculo de secciones sometidas a esfuerzo cortante. De tal forma, con los valores de tensiones residuales a tracción por flexión $f_{R I}$ y $f_{R 3}$ se pueden obtener los valores de resistencia, para el diseño, de piezas de hormigón sometidas a cortante.

Por tal motivo, se estudió el valor de las resistencias de cálculo obtenidas con dos normativas de hormigón estructural: el CM-2010 y la norma española EHE-08 sobre piezas sin armadura de cercos. De tal forma, la EHE-08 considera, de manera aditiva, la contribución de la resistencia del hormigón, el armado longitudinal y la aportación de las fibras, según la ecuación (2). Por su parte el MC-2010 permite calcular la capacidad resistente a cortante mediante la ecuación (3), función de la resistencia del hormigón, de la armadura longitudinal y las fibras.

$$
\begin{gathered}
V_{r d}=\left[\frac{0.18}{\gamma_{c}} \varepsilon\left(100 \rho_{1} f_{c v}\right)^{1 / 3}+0.15 \sigma_{c d}^{\prime}\right] b_{0} d+0.7 \varepsilon \tau_{f d} b_{0} d \\
V_{R d, F}=\left\{\frac{0.18}{\gamma_{c}} k\left[100 \rho_{1}\left(1+7.5 \frac{f_{\text {Ftuk }}}{f_{c t k}}\right) f_{c k}\right]^{1 / 3}+0.15 \sigma_{c p}\right\} b_{w} d
\end{gathered}
$$

Siendo:

$\gamma_{c}$ coeficiente de seguridad del hormigón.

$\varepsilon=k$ factor que toma en consideración el efecto tamaño.

$\rho_{l}$ cuantía de refuerzo de la armadura longitudinal.

$f_{c v}$ resistencia a cortante del hormigón.

$f_{c k}$ resistencia característica a compresión del hormigón.

$\sigma_{c d}^{\prime}=\sigma_{c p}$ tensión por esfuerzo axil.

$b_{0}=b_{w}$ ancho mínimo de la sección.

$d$ canto útil de la sección.

$\tau_{f d}=0,5 f_{c t R, d}=0,33 f_{R, 3, d}$ incremento de resistencia a cortante por efecto de las fibras.

$f_{R 3}$ tensión residual del HRF para un CMOD de 2,5 mm.

$f_{\text {Ftuk }}=f_{R 3} / 3$ tensión residual del HRF.

$f_{c t k}=0,3 f_{c k}^{(2 / 3)}$.

Para poder comparar los resultados analíticos y experimentales fue necesario asumir ciertas consideraciones. Así, el coeficiente de seguridad $\gamma_{c}$ se igualó a la unidad. Para la realización de la comparativa se analizaron los resultados numéricos y experimentales en base a una viga habitual en construcción, tomando el valor de su anchura $b_{0}$ como $300 \mathrm{~mm}$ y su canto útil variable entre 300 y $650 \mathrm{~mm}$. Se prescindió del esfuerzo axil en vigas, por lo que el valor medio de tensión $\sigma_{c d}^{\prime}$ se tomó como cero. Por último, hizo falta considerar un refuerzo con armadura longitudinal de barras de acero por lo que se tomó una cuantía moderada, $\rho_{l}=0,015$.

Con estos condicionantes, además de conocer las resistencias a compresión y residuales a tracción por flexión de los seis hormigones estudiados, fue posible mostrar gráfica y analíticamente la comparativa entre los resultados experimentales y numéricos. De tal forma, primero se presenta la Tabla 3 con los resultados numéricos obtenidos ( $V_{R d}$ según la EHE-08 y $V_{R d, F}$ para el MC-2010) para una viga de dimensiones $300 \times 450 \mathrm{~mm}^{2}$ (ancho-canto útil) frente a los resultados experimentales para deslizamientos a cortante $\left(V_{C S D}\right)$ de 0,5 y $2,5 \mathrm{~mm}$. 
Tabla 3. Valores de cortante $(\mathrm{kN})$ normativo y experimental para una viga de sección 300x450 $\mathrm{mm}^{2}$ (ancho-canto útil).

\begin{tabular}{|c|c|c|c|c|}
\hline & $\boldsymbol{V}_{\boldsymbol{R} d}$ & $\boldsymbol{V}_{\boldsymbol{R d}, \boldsymbol{F}}$ & $\begin{array}{c}\boldsymbol{V}_{\boldsymbol{C S D}} \\
\mathbf{0 , 5}\end{array}$ & $\begin{array}{c}\boldsymbol{V}_{\boldsymbol{C S D}} \\
\mathbf{2 , 5}\end{array}$ \\
\hline SCS50 & 300,3 & 270,9 & 1096,2 & 119,5 \\
\hline SCS70 & 406,7 & 315,9 & 1756,4 & 217,6 \\
\hline VP6 & 159,8 & 171,9 & 375,3 & 140,4 \\
\hline VP75 & 167,1 & 178,2 & 576,5 & 194,4 \\
\hline SCP10 & 255,1 & 243,3 & 351,0 & 155,3 \\
\hline VP10 & 215,9 & 216,0 & 379,4 & 229,5 \\
\hline
\end{tabular}

De manera gráfica, en las Figuras 3(a) a (c) se puede observar la comparativa de resistencias a cortante experimental frente a analítica de tres de los hormigones estudiados. Los tres que no se muestran tuvieron un comportamiento similar a los mostrados.

Analizando los resultados de los hormigones SCS cabe indicar que las resistencias esperadas a cortante según los resultados experimentales para un pequeño deslizamiento $(0,5 \mathrm{~mm})$ son muy superiores a los indicados por las normativas, aumentando linealmente en función del canto útil del elemento. Dichos valores son superiores en más de un 300\% en relación a los valores de cálculo normativos. Sin embargo, al considerar los valores de deslizamiento a cortante de 2,5 mm, los resultados indican que la resistencia a cortante calculada analíticamente supone una resistencia que el material no alcanza.

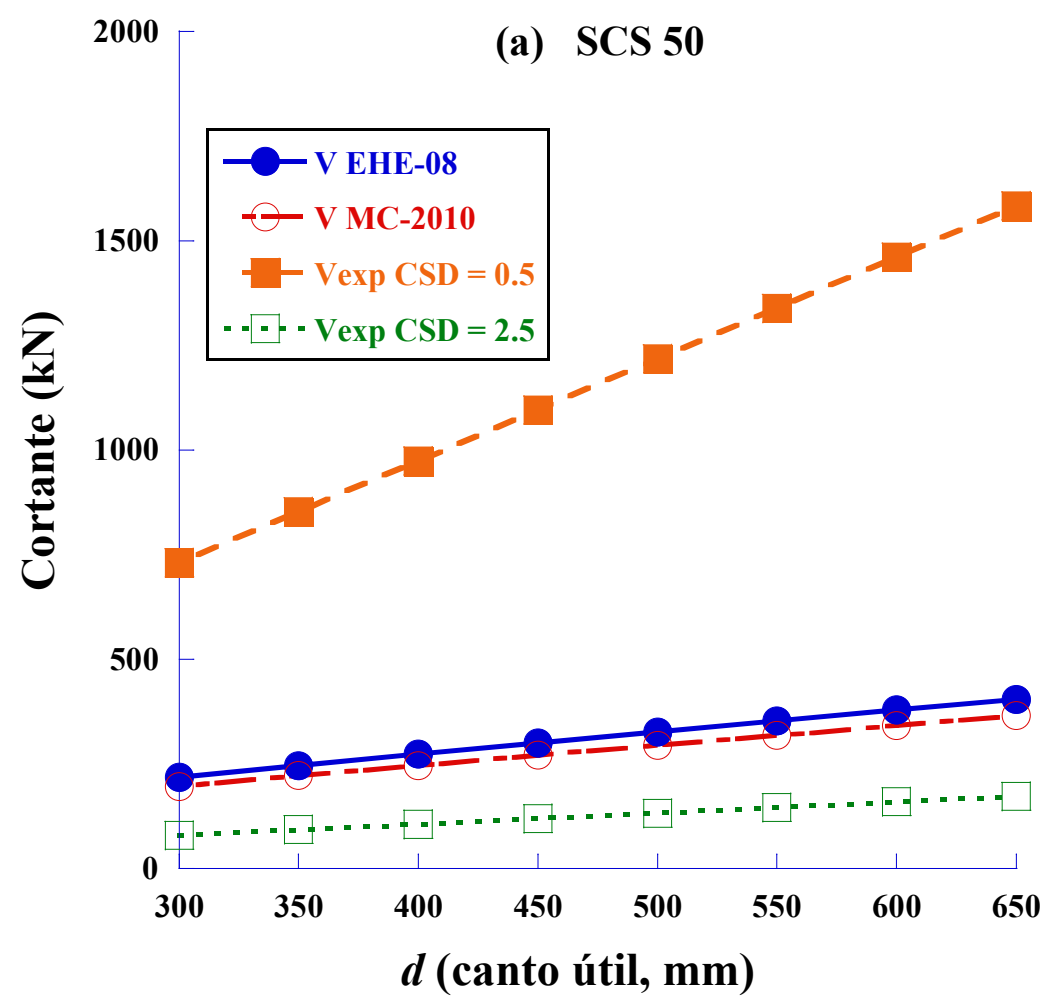

Figura 3(a). Valores de resistencia a cortante obtenidos de manera experimental frente a valores de acuerdo a la formulación normativa para el SCS50. 


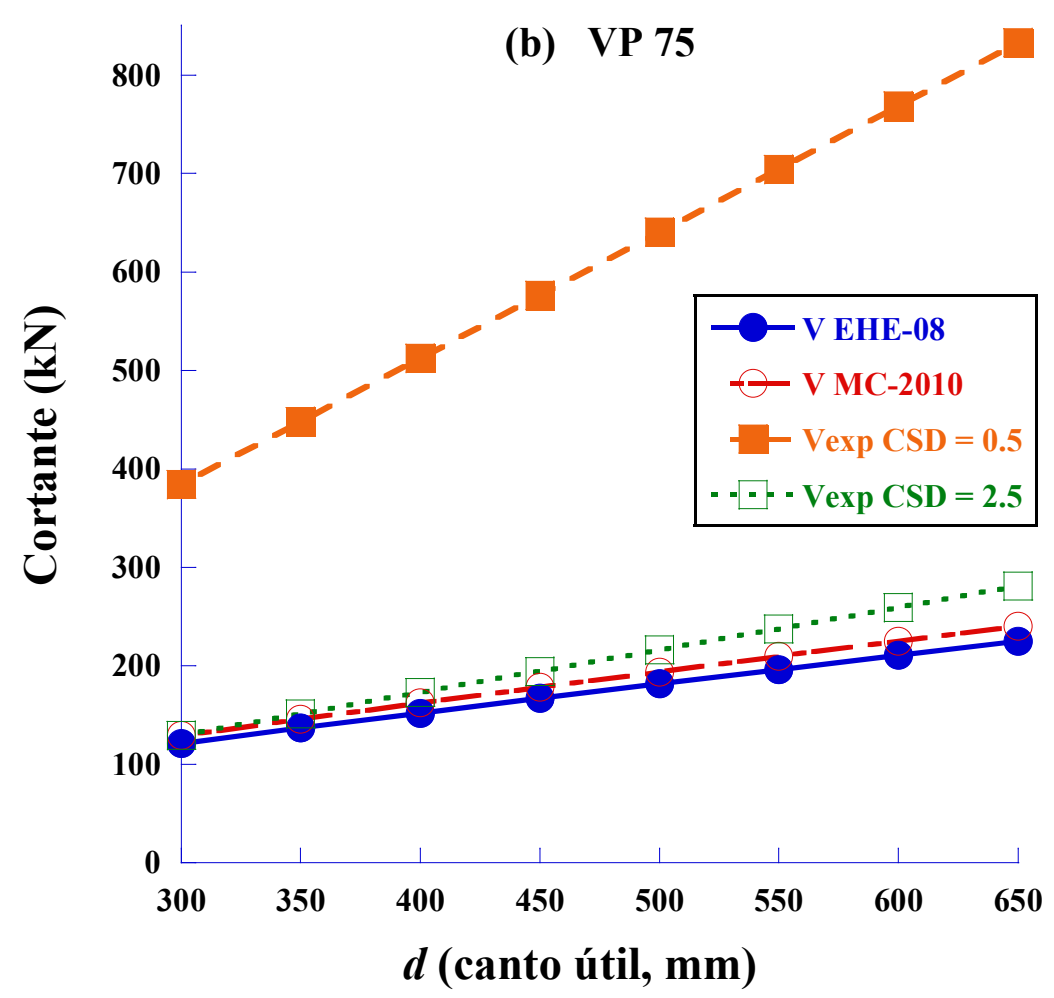

Figura 3(b). Valores de resistencia a cortante obtenidos de manera experimental frente a valores de acuerdo a la formulación normativa para el VP75.

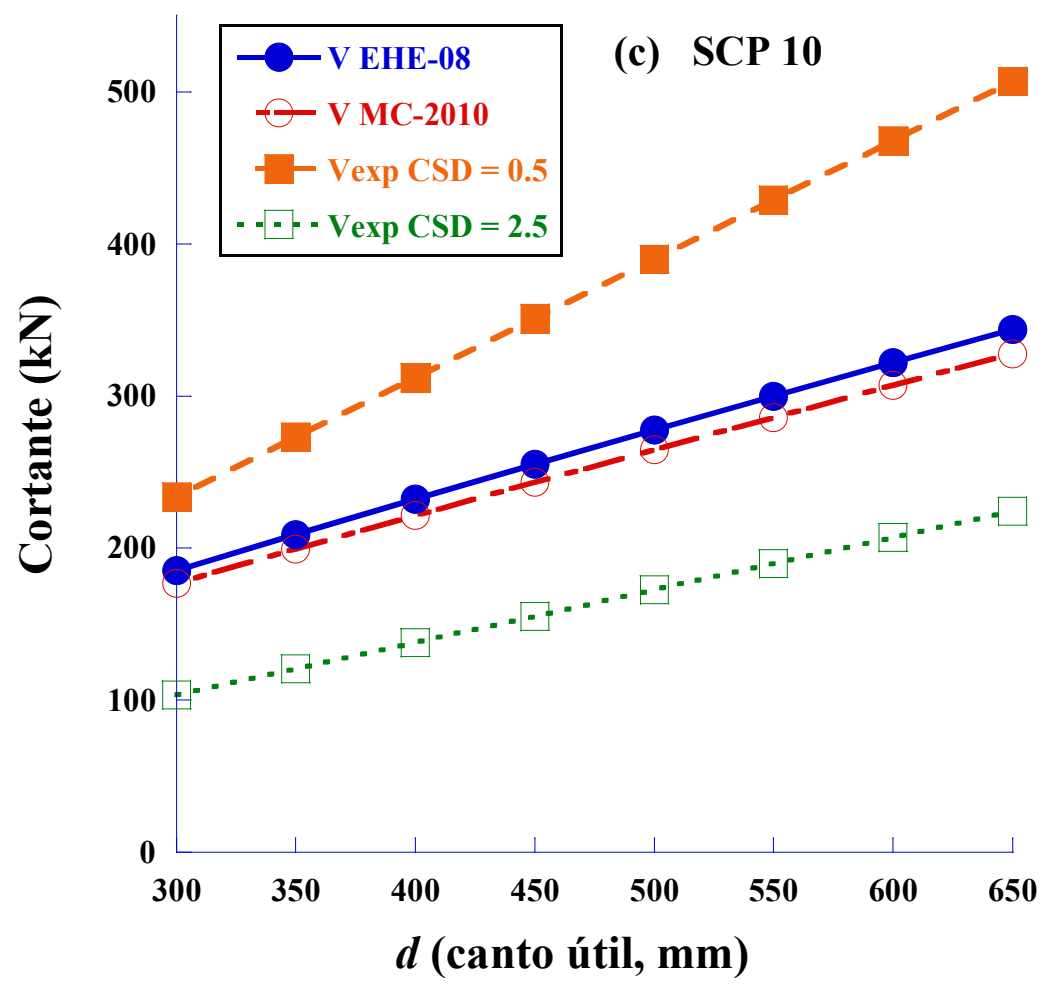

Figura 3(c). Valores de resistencia a cortante obtenidos de manera experimental frente a valores de acuerdo a la formulación normativa para el SCP10. 
En el caso de los dos hormigones de moderada resistencia a compresión también los resultados experimentales para un deslizamiento a cortante de $0,5 \mathrm{~mm}$ son muy superiores a calculado conforme a las normas. Sin embargo, para un deslizamiento mayor los resultados analíticos y experimentales son similares. En el VP6 los valores experimentales se encuentran por debajo de los analíticos, mientras que el VP75 muestra un comportamiento superior al predicho por las normas.

Algo similar a lo expresado para el hormigón VP75 sucede en el VP10. Sin embargo, analizando los resultados del SCP10 su comportamiento se asemeja al de los hormigones reforzados con fibras de acero, todos ellos compactados por vibración. Tal y como se indicó, la orientación de las fibras en las probetas push-off se realizaron con las mitades residuales de ensayos a flexo-tracción, por lo que la orientación de las fibras no es la óptima para soportar esfuerzos cortantes.

\section{CONCLUSIONES}

En relación al esfuerzo cortante en el HRF nos encontramos con la inexistencia de unanimidad a la hora de considerar adecuada la formulación de cálculo indicada por las normativas vigentes. Por tal motivo se realizó el presente estudio sobre HRF con fibras de acero o de poliolefina. Las probetas empleadas, tipo push-off, se obtuvieron mediante mecanizado de las mitades resultantes de probetas ensayadas previamente a flexo-tracción, por lo que se puede decir que se consiguió un estudio mecánico bastante completo del comportamiento de los diferentes hormigones. Las conclusiones más destacadas son:

- Los valores de máxima resistencia a cortante se encuentran asociados a la matriz de hormigón en el HRF con fibras de poliolefina. Sin embargo, si el refuerzo se realiza con fibras de acero pueden observarse mejoras en la resistencia máxima en relación a un hormigón sin reforzar.

- Un incremento de fibras de acero de $20 \mathrm{~kg} / \mathrm{m}^{3}$ (SCS70 frente a SCS50) puede suponer un aumento de un $42 \%$ en la resistencia máxima a cortante.

- Los valores de resistencia residual a cortante se encuentran asociados a la dosificación de las fibras. Para un deslizamiento a cortante de 1,5 $\mathrm{mm}$ la tensión residual que se produjo en el SCS50, con fibras de acero, es equivalente a la del VP6 con fibras de poliolefina, con un valor de 1,4 MPa. Para un deslizamiento a cortante de 1,5 $\mathrm{mm}$ la tensión residual del SCS70 fue superior en un $61 \%$ a la del SCS50 $\left(20 \mathrm{~kg} / \mathrm{m}^{3}\right.$ menos de fibras).

- El comportamiento mostrado por el HRF en los ensayos permite concluir que los procesos de fractura se producen con un incremento importante de la ductilidad y las probetas mostraron la posibilidad de que se produzcan deslizamientos a cortante de hasta $6 \mathrm{~mm}$ sin que se produzca el colapso.

- La comparativa entre los resultados analíticos y experimentales mostraron que las normativas resultan muy conservadoras para deslizamientos a cortante de $0,5 \mathrm{~mm}$. A medida que ese deslizamiento aumenta los resultados experimentales y numéricos son equiparables. Para deslizamientos de $2,5 \mathrm{~mm}$ los resultados experimentales mostraron que, en hormigones autocompactantes elaborados para piezas a flexión, la orientación de las fibras puede no ser la más adecuada para soportar esfuerzos cortantes, por lo que las normas sobrevaloran la contribución de las fibras a la resistencia a cortante. Este fenómeno no se produjo en hormigones compactados por vibración. 


\section{AGRADECIMIENTOS}

Los autores agradecen el apoyo financiero prestado por el Ministerio de Economía y Competitividad de España a través del Proyecto PID2019-108978RB-C31. También agradecen la contribución de Sika SAU a través de la Cátedra Universidad-Empresa "Cátedra Sika-UPM".

\section{REFERENCIAS}

Alberti MG, Enfedaque A, Gálvez JC. Comparison between polyolefin fibre reinforced vibrated conventional concrete and self-compacting concrete. Construction and Building Materials. 2015;85:182-94.

Alberti MG, Enfedaque A, Gálvez JC, Pinillos L. Structural Cast-in-Place Application of Polyolefin Fiber-Reinforced Concrete in a Water Pipeline Supporting Elements. Journal of Pipeline Systems Engineering and Practice. 2017;8(4):05017002.

Alberti MG, Enfedaque A, Gálvez JC, Álvarez C. Using Polyolefin Fibers with Moderate-Strength Concrete Matrix to Improve Ductility. Journal of Materials in Civil Engineering. 2019;31(9):04019170.

Bencardino F, Rizzuti L, Spadea G, Swamy R. Experimental evaluation of fiber reinforced concrete fracture properties. Composites Part B: Engineering. 2010;41(1):17-24.

Cabo AD, Lázaro C, Ros PS. Construcción de la JCHYPAR, una lámina delgada de hormigón reforzado con fibras de acero, en el oceanográfico de Valencia. Hormigón y acero. 2003;228:17786.

Conforti A, Trabucchi I, Tiberti G, Plizzari GA, Caratelli A, Meda A. Precast tunnel segments for metro tunnel lining: A hybrid reinforcement solution using macro-synthetic fibers. Engineering Structures. 2019;199:109628.

Conforti A, Ortiz-Navas F, Piemonti A, Plizzari GA. Enhancing the shear strength of hollow-core slabs by using polypropylene fibres. Engineering Structures. 2020;207:110172.

Cuenca E, Echegaray-Oviedo J, Serna P. Influence of concrete matrix and type of fiber on the shear behavior of self-compacting fiber reinforced concrete beams. Composites Part B: Engineering. 2015;75:135-47.

Dev A, Chellapandian M, Prakash SS. Effect of Macrosynthetic and Hybrid Fibers on Shear Behavior of Concrete Beams Reinforced with GFRP Bars. Journal of Bridge Engineering. 2020;25(7):04020031.

di Prisco M, Plizzari G, Vandewalle L. Fibre reinforced concrete: new design perspectives. Materials and Structures. 2009;42(9):1261-81.

European Committee for Standardization C. Test Method for Metallic Fiber Concrete. Measuring the Flexural Tensile Strength (Limit of Proportionality (LOP), Residual); EN14651:2007+A1. 2007. 
International Federation for Structural Concrete (fib). The fib Model Code for Concrete Structures 2010. Lausanne, Switzerland: International Federation for Structural Concrete; 2010.

Krassowska J, Kosior-Kazberuk M, Berkowski P. Shear behavior of two-span fiber reinforced concrete beams. Archives of Civil and Mechanical Engineering. 2019;19(4):1442-57.

López JA, Serna P, Camacho E, Coll H, Navarro-Gregori J. First ultra-high-performance fibrereinforced concrete footbridge in Spain: design and construction. Structural Engineering International. 2014;24(1):101-4.

Ministerio de Fomento MF. Instrucción de hormigón estructural. EHE-08, 2008.

Ortiz-Navas F, Navarro-Gregori J, Leiva G, Serna P. Comparison of macrosynthetic and steel FRC shear-critical beams with similar residual flexure tensile strengths. Structural Engineering and Mechanics. 2020;76(4):491-503.

Picazo Á, Gálvez JC, Alberti MG, Enfedaque A. Assessment of the shear behaviour of polyolefin fibre reinforced concrete and verification by means of digital image correlation. Construction and Building Materials. 2018;181:565-78.

Picazo Á. Estudio experimental y numérico del comportamiento a cortante de hormigones reforzados con fibras de poliolefina y acero: Ph. D. Thesis, Technical University Madrid, Madrid, Spain.; 2020.

Yoo D-Y, Yuan T, Yang J-M, Yoon Y-S. Feasibility of replacing minimum shear reinforcement with steel fibers for sustainable high-strength concrete beams. Engineering Structures. 2017;147:207-22.

You Z, Chen X, Dong S. Ductility and strength of hybrid fiber reinforced self-consolidating concrete beam with low reinforcement ratios. Systems Engineering Procedia. 2011;1:28-34. 